\title{
Cinética de transformación de la leche a yogur natural usando Lactobacillusjohnsonii(La1) y Streptococcusthermophillus
}

\author{
Alexander Trejo ${ }^{* a}$ Susana $\operatorname{Vargas}^{\mathrm{b}}$, Angel Hernández ${ }^{\mathrm{b}}$, Rogelio Rodríguez*$^{* \mathrm{~b}}$ \\ ${ }^{a}$ Universidad del Valle de México, Ciencias de la Salud, Campus Querétaro, Blvd. Villas del Mesón No. 1000 Col. Provincia \\ JuriquillaC.P. 76230 Querétaro. México. \\ ${ }^{b}$ Universidad Nacional Autónoma de México, Campus Juriquilla, CP 76230. Querétaro, México,
}

*E-mail: lopez_ax1@hotmail.com,rogelior@unam.mx

Recibido 16 agosto 2013, Aceptado 09 octubre 2013

\section{Resumen}

El yogur es un producto lácteo fermentado muy popular, consumido ampliamente en todo el mundo. Este se produce habitualmente por el proceso de fermentación de la leche utilizando dos tipos de bacterias lácticas: Streptococcusthermophillusy Lactobacillusdelbrueckiibulgaricus (St-Lb). En este trabajo se utilizó un cultivo iniciador comercial diferente: el probióticoLactobacillusjohnsonii (Lal) junto con Streptococcusthermophillus (Lal-St) para iniciar el proceso de fermentación. La fermentación de la leche por bacterias lácticas produce cambios morfológicos, químicos y de textura, el objetivo es estudiar la cinética de crecimiento de dos cultivos iniciadores distintos (agregación y cambios estructurales) en la transformación de leche a yogur cambiando la temperatura de incubación y la cantidad de inoculo en el sistema, utilizando las técnicas de caracterización: Dispersión de luz dinámica y Viscosidad, así como los cambios químicos estructurales mediante la espectroscopia Raman, observando las diferencias entre ambos sistemas $(S t-L b)$ y (Lal-St).Se prepararon muestras con tres concentraciones diferentes de cultivo iniciador a diferentes temperaturas de incubación. Los resultados indican la presencia de dos regímenes: el primero (metabolismo primario) se caracteriza por un incremento en el número inicial de bacterias hasta alcanzar una alta concentración de acuerdo con las condiciones de nutrientes presentes, temperatura y espacio disponible; junto con esto dan inicio algunas transformaciones químicas como la de lactosa en ácido láctico y la de fosforo inorgánico en fosfato. El segundo régimen (metabolismo secundario) corresponde propiamente al proceso de fermentación en donde se terminan las transformaciones químicas, se producen los exopolisacaridos y se alcanza la textura final. Los resultados muestran que el uso de este nuevo cultivo da un retraso en la cinética de agregación en comparación con el tradicional.

Palabras clave: Bacterias lácticas; Raman; Dispersión de Luz Dinámica.

\section{Introducción}

El yogur es un producto lácteo fermentado obtenido mediante la transformación de la lactosa en ácido láctico, la cual se lleva a cabo por la acción de bacterias lácticas del cultivo iniciador, que juegan un papel importante durante la producción de yogur en el sabor y la textura[1].

Las bacterias lácticas están ampliamente distribuidas en la naturaleza y la leche es un medio nutricionalmente rico que apoya el crecimiento de muchos microorganismos. La transformación de la leche proporciona control sobre el crecimiento bacteriano necesario para alcanzar un producto deseable [2-7].

El yogur es el producto lácteo fermentado más consumido en el mundo y por esto se ha ganado gran reconocimiento internacional, ya que es un buen vehículo para llevar altas concentraciones de probióticos, con gran eficiencia, al cuerpo humano y que estos lleguen activos al intestino: los beneficios saludables de los probióticos se obtienen solamente cuando estos microorganismos logran llegar vivos y activos al intestino. [8]

La definición de probiótico es "un suplemento alimenticio microbiano vivo que es beneficioso para la salud". Sin embargo, la microflora intestinal tanto en niños como en adultos es muy compleja y se han identificado muchas especies bacterianas. Es evidente que estas especies bacterianas tienen que resistir las condiciones ácidas y las sales biliares que hay enel estómago y alcanzar el intestino [9].

La función de los probióticos puede ser seguida a través del efecto que tienen en los parámetros físicos y químicos: Por ejemplo, los cambios en los perfiles morfológicos y estructurales pueden ser debidos a una posible interacción entre el inóculo y el probiótico [10$11]$.

Durante la fermentación, se produce la ruptura de las proteínas de la leche lo cual aumenta la viscosidad y se producen metabolitos bacterianos que contribuyen al sabor. Durante la acidificación de la leche se forma un gel, debido a la desestabilización de las micelas de caseína y a la acción de nuevas estructuras moleculares producidas por las bacterias: los exopolisacáridos. La formación del gel es una de las propiedades más importantes de los productos de yogurt [12-13].

EL probióticoLal ha sido ampliamente usado en los últimos años para aliviar los problemas gástricos que afectan a más del $50 \%$ de la población humana causada principalmente por la infección con Helicobacter pylori $(\mathrm{Hp})$; este penetra en el revestimiento mucoso del estómago produciendo gastritis aguda y crónica, dolor abdominal, náuseas, úlceras gástricas y duodenales, cáncer de estómago, etc. Lal puede ayudar a las membranas mucosas, y además reduce el riesgo de desarrollar úlceras estomacales causadas por $H p$ [14]. 
Durante la preparación del yogur, la leche sufre agregación importante y cambios estructurales, los cuales dependen de los cultivos y de parámetros como la temperatura y la concentración de arranque.La técnica de dispersión de luz dinámica (DLS) se utiliza para determinar la distribución de tamaños de partículas disueltas o suspendidas en un líquido.La espectroscopia micro-Raman permite obtener información sobre la estructura química de las moléculas, permitiendo obtener la cinética de la modificación química[15].

\section{Parte experimental}

Se uso como cultivo iniciador una muestra comercial (Gastro-Protect TM, Nestlé) que contiene Lactobacillus johnsonii (La1) y Streptococcus thermophillus (St) (La1St). El yogur sin sabor se preparo mezclando el cultivo iniciador con leche comercial (Leche Querétaro, México) pasteurizada entera( $4 \%$ de grasa).Se prepararon muestras a dos temperaturas de incubación 45 y $50{ }^{\circ} \mathrm{C}\left( \pm 0,5^{\circ} \mathrm{C}\right)$, $\mathrm{y}$ tres concentraciones de cultivo iniciador: $\mathrm{C} 1=$ $1 \mathrm{~g} / 100 \mathrm{~mL}\left[1 \times 10^{8} \mathrm{UFC} / \mathrm{g}\right] ; \mathrm{C} 2=2 \mathrm{C} 1 ; \mathrm{C} 3=3 \mathrm{C} 1$.

Un litro de leche se distribuyó por igual en tres reactores de vidrio de $500 \mathrm{ml}$ equipados con termómetro, medidor de $\mathrm{pH}$ y sistema de agitación y el cultivo iniciador correspondiente (Lal-St). Estos tres reactores se colocaron en un baño de temperatura controlada a 45 ${ }^{\circ} \mathrm{C}$. Igual procedimiento se realizó para la otra temperatura.

Se tomaron muestras de $3.6 \mathrm{ml}$ de los reactores cada 20 min durante los primeros $100 \mathrm{~min}$ y $10 \mathrm{~min}$ el tiempo restante de la fermentación para determinar: el tamaño de partícula, la concentración del número de partículas, el $\mathrm{pH}$, la viscosidad y los espectros Raman; este procedimiento se repitió para todas las temperaturas y concentraciones.

Para la determinación del tamaño de partícula y la concentración número de partículas se diluyó una gota $(0.1 \mathrm{ml})$ en $5 \mathrm{ml}$ de agua destilada y se colocó en un un equipo de dispersión de luz. Se utilizaron $0,5 \mathrm{ml}$ de la muestra para obtener la caracterización química usando espectroscopia $\mu$-Raman con fuente de luz a $785 \mathrm{~nm}$; las muestras fueron tomadas cada 20 minutos y se colocaron en un portaobjetos de metal.

El resto de la muestra $(3,0 \mathrm{ml})$ se uso para medir la viscosidad, utilizando un viscosímetro capilar Ubbelohde (Cannon Instr. Co., modelo 0B, PA, EE.UU) calibrado con agua destilada a $25{ }^{\circ} \mathrm{C}$.La viscosidad se puede utilizar para determinar la fracción de volumen de sólidos $(\eta)$ suspendidas en un líquido a través de la relación de Einstein para la viscosidad:

$$
\eta=\eta_{\mathrm{o}}\left(1+\frac{2}{5} \phi\right)
$$

donde $\eta_{\mathrm{o}}$ es la viscosidad del agua. Esta técnica permite caracterizar el proceso de agregación y la aparición de nuevas estructuras (Exopolisacaridos )(EPS) que se producen durante la fermentación: la pendiente de la viscosidad como una función del tiempo $\left(\frac{d \eta}{d t}\right)$ proporciona una medida proporcional a la velocidad de agregación incluyendo la tasa de aparición de EPS durante la reacción:

$$
\frac{d \eta}{d t}=\frac{2 \eta_{o}}{5} \frac{d \phi}{d t}
$$

donde $\phi$ incluye la fracción de volumen de los agregados $\mathrm{y}$ de los EPS. Con este instrumento es posible determinar el inicio de la etapa final de la producción de yogur, es decir, el instante en el que se producen grandes agregados que con el tiempo, forman un gel suave característico del yogur (16-18).El pH se determinó directamente en el reactor.

De esta manera fue posible determinar los perfiles morfológicos y estructurales como una función del tiempo de incubación. Estos resultados se compararon con los obtenidos en las mismas condiciones de temperatura y concentración para los sistemas con el cultivo iniciador habitual que es $L b-S t$.

El proceso de incubación se detuvo cuando el tamaño de los agregados fue tan grande (pocas micras) que ya no se podían medir por dispersión de luz, lo que corresponde a los tiempos de entre 150 y 170 minutos dependiendo de la temperatura y la concentración inicial del cultivo.

\section{Resultados y discusión}

Los perfiles de agregación se muestran en las Figs. 1a y $1 \mathrm{~b}$ a 45 y $50{ }^{\circ} \mathrm{C}$ respectivamente para todas las concentraciones; estos perfiles se presentan junto con los correspondientes al cultivo iniciador $(L b-S t)$ a efectos de comparación.

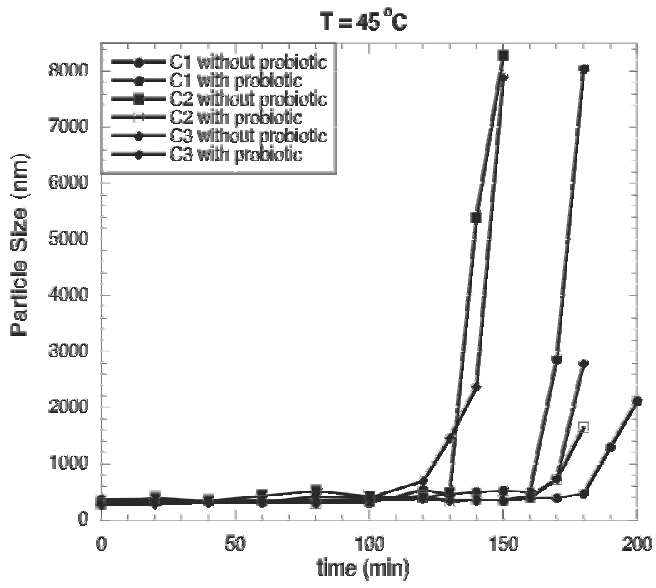

Figura 1a. Tamaño de partícula de las muestras con y $\sin$ probiótico. 


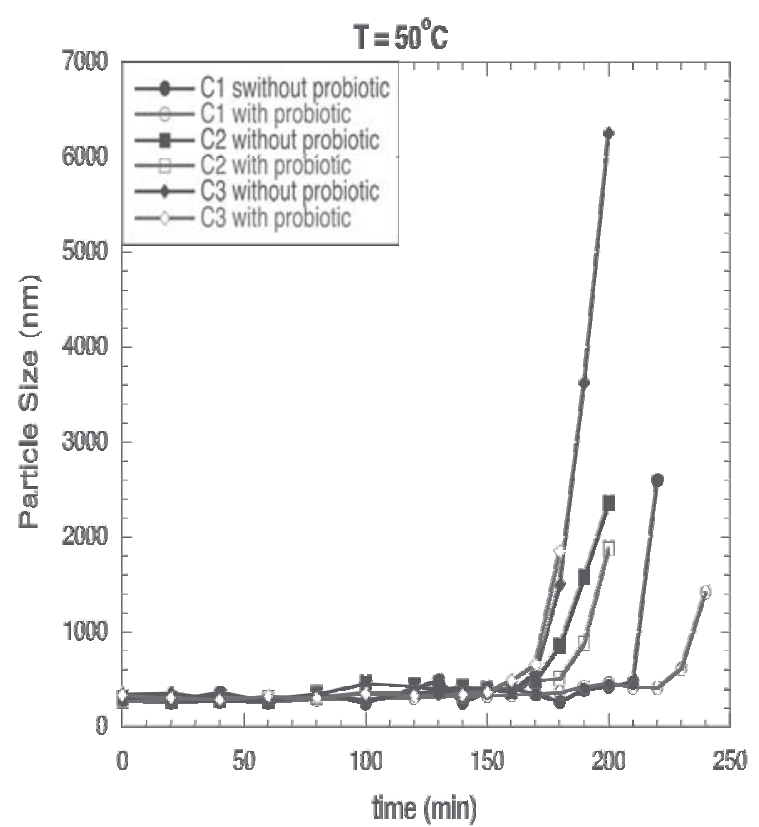

Figura 1b. Tamaño de partícula de las muestras con y sin probióticos

En la figuras anteriores es posible observar que en todos los casos, los perfiles muestran dos regímenes: El primero corresponde al metabolismo primario el cual se caracteriza por un tamaño de partículaconstante con tamaños promedio de $320 \mathrm{~nm}$ para $(L b-S t)$ y $290 \mathrm{~nm}$ para (Lal-St).El segundocorresponde al metabolismo secundario y se caracteriza por un incremento abrupto en el tamaño de partícula correspondiente a la formación de grandes agregados y de los exopolisacáridos (EPS). También se observa que el sistema inoculado con (LalSt) necesita tiempos más largos para alcanzar el metabolismo secundario, en comparación con el sistema inoculado con $(L b-S t)(1)$.

Durante el primer régimen, el metabolismo primario y el tamaño de partícula permanecen constantes, Cuando se alcanza el segundo régimen, el proceso de fermentación acelera los cambios físicos y químicos que crean estructuras nuevas.

Para el sistema que contiene los cultivos $(L b-S t)$, el tiempo promedio es de de $135 \mathrm{~min}$, mientras que para el sistema Lal el tiempo promedio es de $175 \mathrm{~min}$, es decir $30 \%$ más.La tasa de crecimiento para el sistema ( $\mathrm{Lal}-\mathrm{St}$ ) es baja ya que toma tiempos más largos para llegar a producir el yogur. En el sistema $(L b-S t)$, hay una mejor simbiosis entre las bacterias lo que provoca una alta tasa de crecimiento de estas: en los primeros tiempos de incubación, $L b$ estimula el crecimiento de $S t$, siendo éste el que inicialmente reduce el $\mathrm{pH}$ por la producción de ácido láctico;luego, cuando la concentración de $L b$ ha crecido, es esta bacteria la principal productora de ácido para reducir el $\mathrm{pH}$ a 4.5 al final de la fermentación (1).La dependencia de la viscosidad al tiempo de incubación se muestra en las figuras $2 \mathrm{a}$ y $2 \mathrm{~b}$

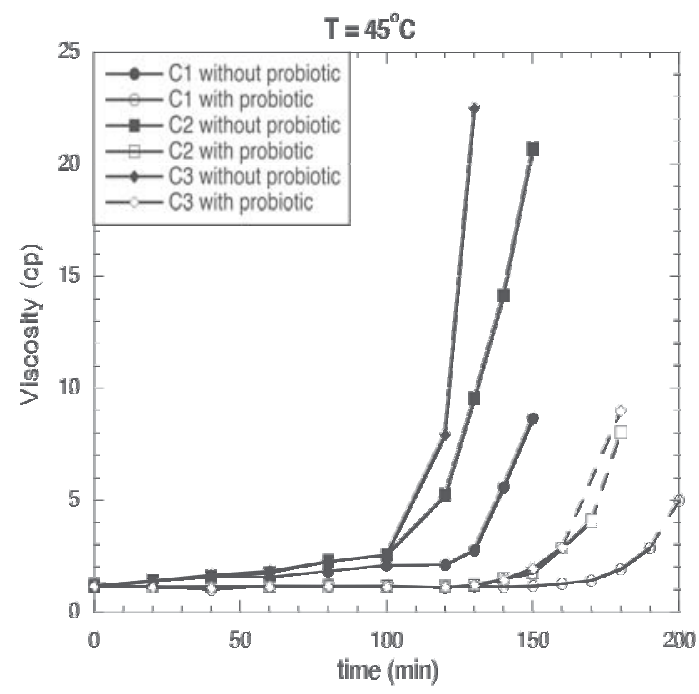

Figura 2a. Perfiles de viscosidad para muestras con y sin probióticos.

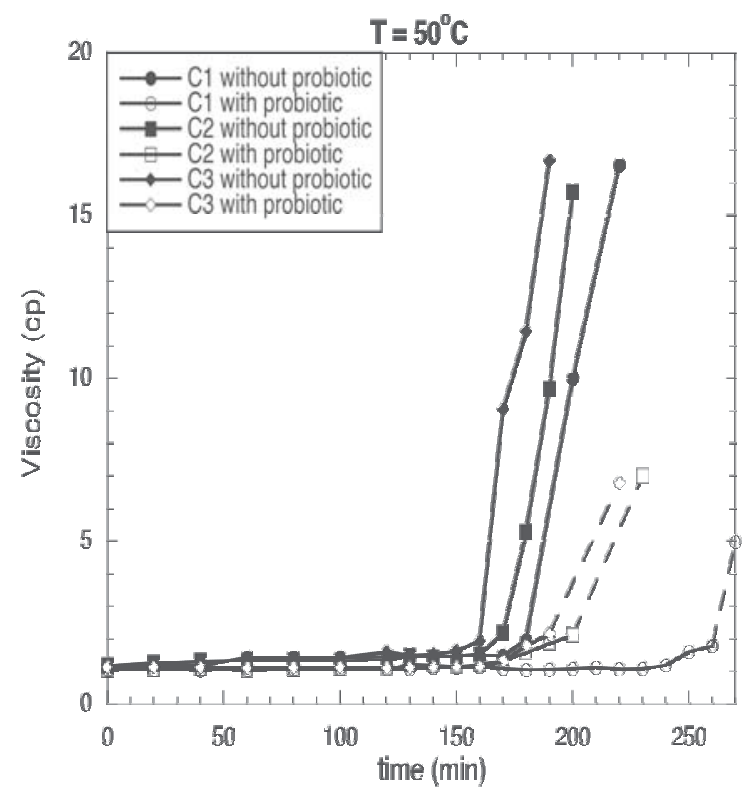

Figura 2b. Perfiles de viscosidad para muestras con y sin probióticos.

En la figuras $2 \mathrm{a}$ y $2 \mathrm{~b}$ se puede observar que durante el metabolismo primario la viscosidad se incrementa linealmente con el tiempo de incubación. Estos resultados están en completo acuerdo con los resultados obtenidos por Dispersión de Luz mostrados en las Figs. 1a y $1 \mathrm{~b}$. Esta es una de las principales diferencias entre el yogur preparado sin probióticos $(S t-L b)$ y el preparado usando Lal-St . El metabolismo secundario inicia los cambios físicos y químicos importantes, correspondiendo a un proceso complejo en donde además de la producción de ácido láctico que a su vez reduce aún más el $\mathrm{pH}$, las bacterias producen exopolisacáridos (EPS), que aumentan la viscosidad, y le dan la textura final al yogur. 


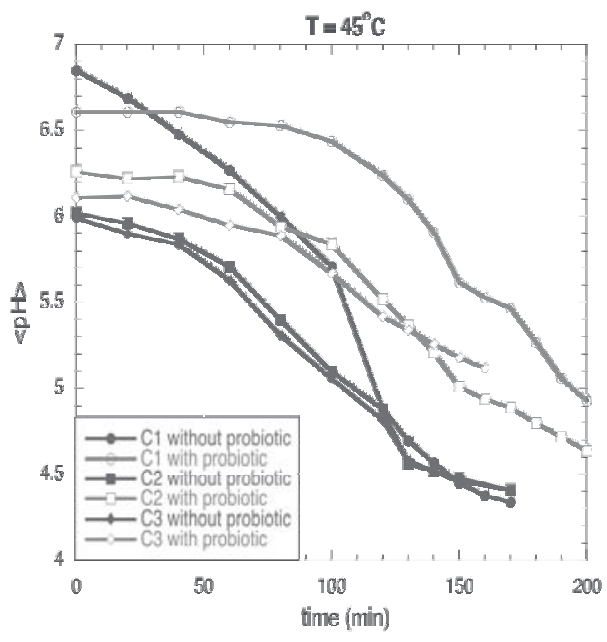

Figura 3a. Perfiles de $\mathrm{pH}$ para muestras con y sin probiótico.

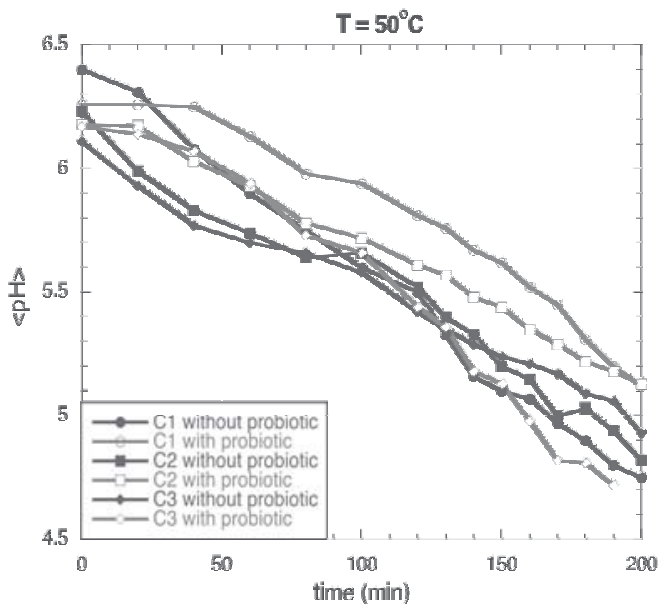

Figura 3b. Perfiles de $\mathrm{pH}$ para muestras con y sin probiótico

En las Figuras $3 \mathrm{a}$ y $3 \mathrm{~b}$ es posible observar la dependencia del $\mathrm{pH}$ con el tiempo para todas las muestras.En todos los casos el $\mathrm{pH}$ se reduce con el tiempo, sin embargo, para el sistema (Lal-St) a baja temperatura $\left(45^{\circ} \mathrm{C}\right)$, el $\mathrm{pH}$ se mantiene prácticamente constante durante 80-100 min; después de este tiempo el $\mathrm{pH}$ se reduce alcanzando valores de entre 4,5 y 5 . A mayor temperatura $\left(50^{\circ} \mathrm{C}\right)$ se acelera la reducción del $\mathrm{pH}$ en todo momento y para todas las muestras. Al final todas las muestras se comportan prácticamente de la misma manera reduciendo el $\mathrm{pH}$ al mismo valor finalentre 4,5 y (1).

Las bacterias lácticas producen la transformación del fósforo inorgánico en orgánico y de la lactosa en ácido láctico. También producen los exopolisacáridos (EPS). La cinética de estas transformaciones se obtuvo a partir de los espectros Raman. La Figura 4a muestra un espectro Raman de Lal-St a $45^{\circ} \mathrm{C}$ y a concentración C2, después de $100 \mathrm{~min}$ de la inoculación. El recuadro muestra un detalle de las bandas en números de onda bajos.

La figura $4 \mathrm{~b}$ muestra, como ejemplo, la evolución temporal de la intensidad de la banda en $222 \mathrm{~cm}^{-1}$; son estos cambios en la altura de las bandas los que permiten obtener información sobre la cinética de la modificación estructural.

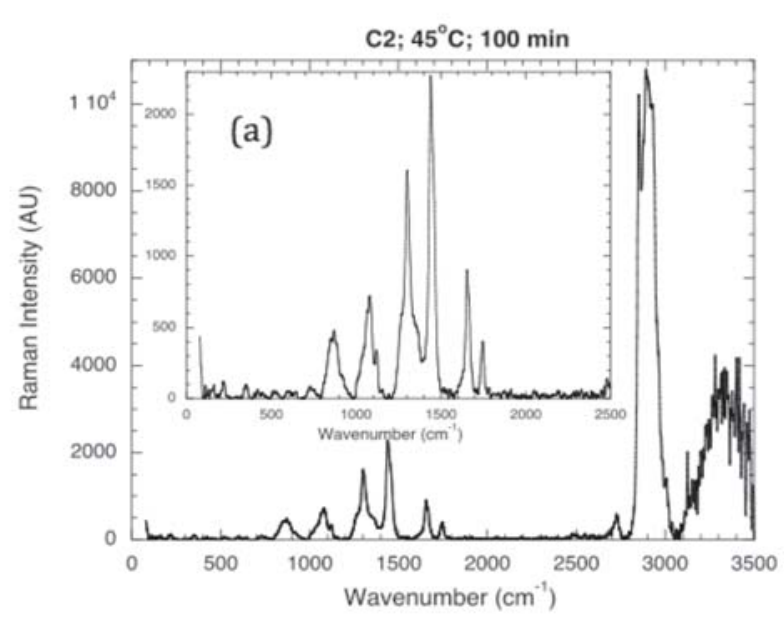

Figura 4a.Espectro Raman de la muestra a una concentración $\mathrm{C} 2$, a $45^{\circ} \mathrm{C}$ y 100 min de tiempo de incubación; se muestran números de onda bajos.

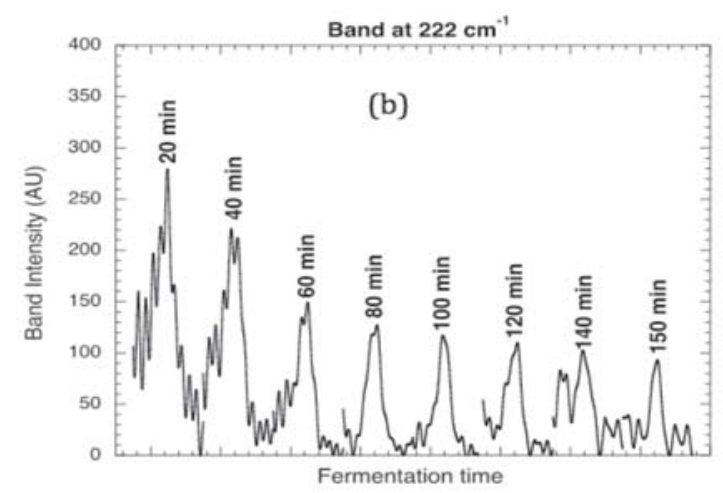

Figura 4b. Altura de las bandas a $222 \mathrm{~cm}^{-1}$ a diferentes tiempos para la concentración $\mathrm{C} 2 \mathrm{a} 45^{\circ} \mathrm{C}$.

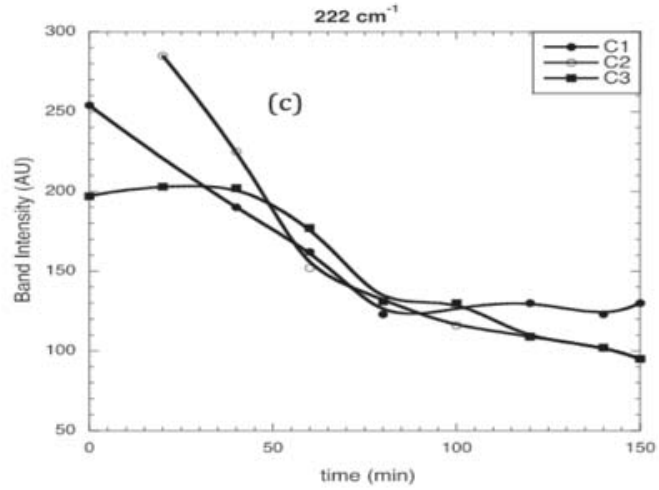

Figura 4c. Perfiles de intensidad de banda para la banda a 222 $\mathrm{cm}^{-1}$ para todas las concentraciones a $45^{\circ} \mathrm{C}$.

En la figura $4 \mathrm{c}$ se muestran los perfiles de intensidad de la banda en $222 \mathrm{~cm}^{-1}$ a $45 \mathrm{C}$ para todas las concentraciones. Esta banda puede ser asignada a la presencia de lactosa en el inicio del proceso de fermentación. La figura muestra que hay una reducción en la intensidad de la banda en el tiempo, lo que significa que la lactosa se está transformando en ácido láctico. 
Como se puede observar, estas bandas del espectro Raman muestran una rápida transformación de la lactosa en ácido láctico durante los primeros minutos del proceso de fermentación;después de aprox. $80 \mathrm{~min}$, la transformación se produce a baja velocidad debido principalmente a una reducción del $\mathrm{pH}$.

\section{Conclusiones}

Se prepararon yogures con diferentes cultivos iniciadores (Lb-St y Lal-St) en las mismas condiciones y se compararon los resultados. La cinética de agregación y la modificación química se obtuvo como una función del tiempo de incubación. Se observaron dos regímenes: El primero corresponde a un metabolismo primario, caracterizado por un tamaño constante de las partículas prácticamente, sin embargo, en este régimen la intensidad dispersada $\mathrm{y}$ la viscosidad aumentan linealmente con el tiempo, lo que indica que el número de bacterias del cultivo inicial crecen con el tiempo porque están en condiciones apropiadas de temperatura y nutrientes.

El segundo régimen, correspondiente al metabolismo secundario, se caracteriza por una rápida reducción en el $\mathrm{pH}$, la producción de EPS, un rápido incremento en el tamaño de partícula debido al proceso de agregación y la formación de una estructura de gel suave característico del yogur.

La principal diferencia entre los dos cultivos iniciadores es que para ( $L b-S t)$ hay una fuerte simbiosis entre ambos tipos de bacterias que producen una alta tasa de crecimiento de las bacterias, mientras que para ( $\mathrm{Lal}$ $S t$ ) no hay simbiosis y hay un retraso en el tiempo requerido para alcanzar el régimen secundario. En ambos casos el $\mathrm{pH}$ se reduce con el tiempo de incubación.

En los espectros Raman se muestran las principales modificaciones estructurales las cuales fueron:

1) Los grupos funcionales de lactosa desaparecen con el tiempo mientras aparece el grupo carboxílico del ácido láctico;

2) La transformación del fosforo inorgánico en fósforo orgánico;

3) La producción de exopolisacáridos.

Esta información es muy importante pues no hay trabajos reportados usando estas técnicas. Concluimos que el consumo de Lal es recomendable pues es un mejor cultivo iniciador en comparación del $L b$ en cuanto a efectos beneficiosos, aunque esperábamos que este nuevo cultivo tuviera una producción más rápida de yogur.

Es importante recalcar que no hay antecedentes previos sobre este tema, lo que realza la importancia de este estudio, pues este trabajo abre la puerta a más investigaciones para la mejora de muchos aspectos de los productos

\section{Referencias}

1. R. Rodríguez, S. Vargas, M. Estévez, F. Quintanilla, Alexander Trejo-Lopez, A.R. Hernández Martínez; Vib. Spectrosc. , 2013, 68, 133-140

2. F.M. Driessen, F. Kingma, \& J. Stadhouders, Neth. Milk Dairy J.1982, 36, 135-140.
3. W. Fu, \& A.P. Mathews, Biochem. Eng. J1977, 3, 163-170.

4. A. Haque, R.K.Richardson\& E.R. Morris, Food Hydrocolloid, 2001,15 (4-6), 593-602.

5. E.W Ng, M. Yeung \& P.S. Tong,Int J Food Microbiol, 2011, 145(1) 169-175.

6. S. Norton, C. Lacroix\& JC. Vuillemard, Enzyme Microb Tech, 1994, 16(6), 457-466.

7. A.Y. Tamime, Some Aspects of the production of yoghurt and condensed yoghurt, $\mathrm{PhD}$ Thesis. University of Reading.1977.

8. Elizabeth W. Ng, Marie Yeung, Phillip S. Tong;Int J Food Microbiol,2010,145, 169-175

9. R. Amores, A. Calvo, J.R. Maestre y D. MartínezHernández; Rev. Esp. Quimioterap, 2004, 17 (No 2): 131139

10. M. Del Piano, L. Morelli, G.P. Strozzi, S. Allesina, M. Barba, F. Deidda, Digest Liver Dis.2006, 38, 248-255.

11. P.J. Jones, K.A. Varady, APNM,2008, 33(1), 118-123.

12. D.D.G. Mater, L. Bretigny, O. Firmesse, M.J. Flores, A. Mogenet, J.L. Bresson\& G. Corthier, FEMS MicrobiolLett.2005, 250, 185-187.

13. T. Vasiljevic\& N.P. Shah, Handbook of Food Product Manufacturing, 2004, 2.593-663

14. Armuzi A, Cremonini F, Ojetti V, Bartolozzi F, Canducci F, Candelli M, Santarelli L, Cammarota G, de Lorenzo A, Pola P, Gasbarrini G \& Gasbarrini A,Digestion, 2001, 63, 1-7.

15. S. Gallier, K.C. Gordon, R. Jimenez-Flores, \& D.W. Everett, Int Dairy J, 2011,21, 402-412.

16. M.R. Cannon, E. Manning, \& J.D. Bell, Anal Chem,1960, 32(3) 355-358

17. D. Jaworska, B. Waszkiewicz-Robak, W. Kolanovski, \& F. Swiderski, Int J DairyTechnol, 2005, 58, 39-40. "

18. A.P. Marafon, A. Sumi, M.R. Alcantara, A.Y. Tamime\& M. Nogueira de Oliveira LWT-FoodSciTechnol. 2011, 44(2) 511-519. 